\title{
GOLPH2, a gene downstream of ras signaling, promotes the progression of pancreatic ductal adenocarcinoma
}

\author{
JUAN DUAN $^{1,2}$, XIAOGUANG LI ${ }^{3}$, SHAN HUANG $^{1}$, YANHUA ZENG $^{1}$, YAN HE $^{1}$, \\ HEKUN LIU ${ }^{2}$, DEXIN LIN ${ }^{1}$, DONGDONG LU ${ }^{3}$ and MIN ZHENG ${ }^{1,2}$ \\ ${ }^{1}$ Department of Biochemistry and Molecular Biology, School of Basic Medical Sciences; \\ ${ }^{2}$ Translational Medicine Institute, Fujian Medical University, Fuzhou, Fujian 350108; \\ ${ }^{3}$ School of Life Science and Technology, Tongji University, Shanghai 200092, P.R. China
}

Received September 24, 2016; Accepted June 16, 2017

DOI: $10.3892 / \mathrm{mmr} .2018 .8430$

\begin{abstract}
Various studies have previously demonstrated that Golgi protein-73 (GOLPH2) is overexpressed in tumorigenesis, which has been observed in hepatocellular carcinoma and prostate cancer. However, the expression levels and specific functions of GOLPH2 in the progression of pancreatic cancer remain to be elucidated. The present study aimed to investigate the expression of GOLPH2 in pancreatic ductal adenocarcinoma (PDAC) tissues and examined the effects of GOLPH2 on the growth and migration of pancreatic cancer cells. In the present study, the mRNA levels of GOLPH2 in PDAC cancer tissues were examined using RT-qPCR. The effects of GOLPH2 on the growth and migration of cancer cells were examined using crystal violet and Boyden chamber assays. The study demonstrated that the expression of GOLPH2 mRNA and protein was elevated in PDAC clinical tissues. The growth and motility of the PDAC cells was enhanced following overexpression of GOLPH2, whereas downregulating the expression of GOLPH2 impaired the growth, motility and tumorigenesis. Furthermore, GOLPH2 was observed to interact with protein kinase B (Akt), which subsequently increased the activity of Akt. In addition, GOLPH2 was revealed as a downstream gene of Ras signaling and promoted the transformation of normal pancreatic cells. The results of the present study revealed the important functions of GOLPH2 in PDAC, and suggest that GOLPH2 may act as a promising therapeutic target for the treatment of PDAC in the future.
\end{abstract}

Correspondence to: Dr Juan Duan, Department of Biochemistry and Molecular Biology, School of Basic Medical Sciences, Fujian Medical University, 1 Xueyuan Road, Fuzhou, Fujian 350108, P.R. China E-mail: duanjuan0723@aliyun.com

Key words: Golgi protein-73, pancreatic ductal adenocarcinoma, cell growth, cell motility, protein kinase B

\section{Introduction}

Clinical progression regarding pancreatic ductal adenocarcinoma (PDAC) has been made in previous years, however the 5 -year survival rate is still $<10 \%$ (1). Due to the limited investigations of the underlying molecular mechanisms and the lack of sensitive and specific biomarkers for early diagnosis, the prognosis and diagnosis for this disease are very poor. Therefore, identification of the molecular mechanisms underlying PDAC are of primary concern and will benefit the clinical treatment for patients.

Golgi protein-73 (GOLPH2) was firstly identified as a type II Golgi glycoprotein (2). The expression of GOLPH2 is predominantly observed in the epithelial cells of biliary, gut, stomach and prostate (3). Aberrant expression of GOLPH2 has been detected in various diseases. The pathological process underlying the progression from hepatitis and cirrhosis to hepatocellular carcinoma (HCC), reveals a gradual increase in the expression levels of GOLPH2 (4). Furthermore, the high expression of GOLPH2 in the serum of HCC patients is considered a novel biomarker for the development of the disease (4). In addition, the cancerous tissues of non-small cell lung, renal cell and prostate cancers demonstrate increased expression of GOLPH2 (5-7). In addition, GOLPH2 has been reported to regulate the immune response (8). However, the expression pattern and the biological functions of GOLPH2 in PDAC remain unknown.

Activation of protein kinase B (Akt) signaling is typical in pancreatic cancer. Akt signaling is important in the growth and migration of pancreatic cancer cells (9-11). Akt is an effector downstream of the growth factor receptor, therefore amplification of growth factor receptor leads to the activation of Akt. Previously, activation of Akt has been observed in the Golgi apparatus. GOLPH3 recruits Akt to the Golgi apparatus and is responsible for its subsequent activation (12). However, the exact molecular mechanisms underlying the activation of Akt in the Golgi apparatus remain to be fully elucidated.

The present study investigated the expression profile and the biological functions of GOLPH2 in pancreatic cancer, and aimed to elucidate the underlying molecular mechanisms responsible for the progression of the disease. 


\section{Materials and methods}

Cell culture. Pancreatic cancer cells (Suit2, BXPC3), normal pancreatic cell line HPDE, mouse fibroblast NIH3T3 and human embryonic HEK293T cells were incubated in Dulbecco's modified Eagle's medium (Invitrogen; Thermo Fisher Scientific, Inc. Waltham, MA, USA) supplemented with $10 \%$ fetal bovine serum (Sigma-Aldrich; Merck KGaA, Darmstadt, Germany). Cells were cultured in an incubator at $37^{\circ} \mathrm{C}$, in an environment containing $5 \% \mathrm{CO}_{2}$.

PDAC samples. The present study was approved by the ethics committee of Fujian Medical University (Fuzhou, China) and written informed consent was obtained from all patients (age, 50-60; 38 male and 14 female; all patients exhibited PDAC, and patients who had received chemotherapy were excluded from the study). A total of 52 PDAC tissues and paired non-cancerous tissues were obtained from the affiliated hospital of Fujian Medical University and were stored at $-80^{\circ} \mathrm{C}$.

Plasmids and establishment of stable cell lines. The DNA insert encoding GOLPH2 was cloned into the plasmid pcDNA 3.1-myc (Clontech Laboratories, Inc., Mountainview, CA, USA). Plasmids ( $1 \mu \mathrm{g})$ were transfected into BXPC3 and Suit2 cells using PolyJet (SignaGen, Rockville, MD, USA) according to the manufacturer's protocols. Cells were selected with G418. The expression of exogenous GOLPH2 was confirmed by western blotting using the myc tag.

Reverse transcription-polymerase chain reaction ( $R T-q P C R)$. The RNA was extracted using TRIzol (Invitrogen; Thermo Fisher Scientific, Inc.) according to the manufacturer's protocols. The reverse transcription was performed according to the manufacturer's protocol (Promega Corporation, Madison, WI, USA), qPCR was conducted in a Bio-Rad PCR system (Bio-Rad Laboratories, Inc., Hercules, CA, USA) with a qPCR 2X SYBR Green Mixture (Takara Biotechnology Co., Ltd., Dalian, China) according to the manufacturer's protocol with the following conditions: $95^{\circ} \mathrm{C}$ for $30 \mathrm{sec}$, followed by 40 cycles of $95^{\circ} \mathrm{C}$ for $5 \mathrm{sec}$ and $60^{\circ} \mathrm{C}$ for $30 \mathrm{sec}$. The primer sequences used were as follows: Forward, 5'-TGGAGAGCG TCAACA AGCTG-3' and reverse, 5'-GACATCCTGCTA GCCTGC-3' for human GOLPH2 gene; forward, 5'-GAT CATTGCTCCTCCTGAGC-3' and reverse, 5'-ACTCCT GCTTGCTGATCCAC-3' for human $18 \mathrm{~S}$ which served as an internal control. Dissociation was performed at the end of the procedure to confirm that non-specific amplification had not occurred. The relative levels of gene expression were calculated using the $2^{-\Delta \Delta C q}$ method (13).

Western blot analysis. Cultures were harvested and the protein was extracted using a radioimmunoprecipitation assay buffer (Cell Signaling Technology, Inc., Danvers, MA, USA) containing protease and phosphatase inhibitors. The concentration of proteins was determined using the Bradford assay. Proteins (15 $\mu \mathrm{g} /$ lane) were separated by $10 \%$ SDS-PAGE, transferred onto polyvinylidene difluoride membranes (EMD Millipore, Billerica, MA, USA) and probed with specific primary antibodies at $4{ }^{\circ} \mathrm{C}$ overnight and the secondary antibodies at room temperature for $1 \mathrm{~h}$. The immunoreactive protein was examined using an enhanced chemiluminescence kit (Pierce; Thermo Fisher Scientific, Inc.). GOLPH2 antibody (cat. no. Ab109628; 1:1,000) was obtained from Abcam (Cambridge, MA, USA). Akt (cat. no. 4685; 1:1,000), Cyclin D1 (cat. no. 2978; 1:1,000), B-cell lymphoma (Bcl)-2 (cat. no. $15071 ; 1: 1,000$ ) and Bcl-2 associated $X$ (cat. no. 2764; 1:1,000), apoptosis regulator (Bax; cat. no. 5023; 1:1,000) antibodies were obtained from Cell Signaling Technology, Inc., (Danvers, MA, USA) and the GAPDH antibody (cat. no. sc-25778; 1:5,000) was obtained from Santa Cruz Biotechnology (Dallas, TX, USA). HRP linked secondary antibodies (anti-mouse IgG; cat. no. 7076 and anti-rabbit IgG; cat. no. 7074) were obtained from Cell Signaling Technology, Inc. and used at 1:2,000.

GST pull-down assay. The fusion protein GST-AKT was purified using Sepharose4B beads (GE Healthcare Life Sciences, Little Chalfont, UK) according to the manufacturer's protocols. Cell lysates were prepared using the lysis buffer. Following centrifugation $\left(4^{\circ} \mathrm{C}, 10,000 \mathrm{x} \mathrm{g}, 20 \mathrm{~min}\right)$, the supernatant was incubated with $5 \mu \mathrm{g}$ GST fusion protein overnight at $4^{\circ} \mathrm{C}$. The Sepharose $4 \mathrm{~B}$ beads were added and incubated with cell lysate for another $4 \mathrm{~h}$. Then, the beads were washed and the protein pulled down and detected with western blot analysis.

Knockdown of GOLPH2 expression. The commercial available RNAi lenti-virus short hairpin (Sh) con and Sh GOLPH2 were supplied by Shanghai GenePharma Co., Ltd (Shanghai, China). Virus was added to the BXPC 3 and Suit 2 cell culture medium and the transfection was performed according to the manufacturer's protocols (MOI=1). Cells were then selected with puromycine $24 \mathrm{~h}$ following transfection.

Immunoprecipitation assay. The immunoprecipitation method was conducted as previously described (4). Cells were washed with ice-cold PBS and lysed in Tris-buffered saline (pH 7.4), containing $50 \mathrm{mM}$ Tris, $150 \mathrm{mM} \mathrm{NaCl}, 1 \% \mathrm{NP}-40$, $1 \mathrm{mM}$ EDTA, $1 \mathrm{mM}$ Na3VO4, $10 \mathrm{mM} \mathrm{NaF}, 2.5 \mathrm{mg} / \mathrm{ml}$ aprotinin and leupeptin, $1 \mathrm{mM}$ b-glycerophosphate and AEBSF (4-(2-aminoethyl) benzenesulfonyl fluoride hydrochloride) and $10 \mathrm{mM}$ iodoacetate. Lysates were incubated on ice for $15 \mathrm{~min}$ prior to removal of cellular debris and nuclei via centrifugation at $4^{\circ} \mathrm{C}$ and $10,000 \mathrm{xg}$ for $20 \mathrm{~min}$. Cell lysates were incubated with the AKT antibody (cat. no. 4685; 1:1,000; Cell Signaling Technology, Inc.) overnight at $4^{\circ} \mathrm{C}$. Protein A-Sepharose (GE Healthcare Life Sciences) beads in a 50:50 mixture in $50 \mathrm{mM}$ Tris buffer, ( $\mathrm{pH}$ 7.0), were added, and further incubation occurred for another $4 \mathrm{~h}$ at $4^{\circ} \mathrm{C}$. The immunoprecipitates were washed 4 times in Tris-buffered saline and boiled for $5 \mathrm{~min}$ in $40 \mu \mathrm{l}$ Laemmli buffer containing $0.02 \%$ blue bromophenol and $2 \%$ b-mercaptoethanol.

Immunohistochemistry $(I H C)$. Tissues were fixed with $4 \%$ formalin at $4^{\circ} \mathrm{C}$ overnight, embedded in paraffin, cut as $5 \mu \mathrm{m}$-thick consecutive sections and then deparaffinized at room temperature using xylene and ethanol. Antigen recovery was performed in sodium citrate solution $(\mathrm{pH} 6.0,20 \mathrm{~min}$, $95^{\circ} \mathrm{C}$ ). Following this, the sections were washed 3 times with $0.01 \mathrm{~mol} / \mathrm{l} \mathrm{PBS}\left(8 \mathrm{mmol} / 1 \mathrm{Na}_{2} \mathrm{HPO}_{4}, 2 \mathrm{mmol} / 1 \mathrm{NaH}_{2} \mathrm{PO}_{4}\right.$ and $150 \mathrm{mmol} / \mathrm{l} \mathrm{NaCl}$ ) for $5 \mathrm{~min}$ each. They were then blocked for 
$1 \mathrm{~h}$ in $0.01 \mathrm{~mol} / \mathrm{l}$ PBS containing $0.3 \%$ Triton X-100 and 5\% BSA (Shanghai Shenggong Biology Engineering Technology Service, Ltd., Shanghai, China) at room temperature, followed by addition of anti-GOLPH2 (cat. no. Ab109628; 1:100) antibody at $4^{\circ} \mathrm{C}$ for at least $8 \mathrm{~h}$. Following brief washing with $0.01 \mathrm{~mol} / 1 \mathrm{PBS}$, sections were incubated with $0.01 \mathrm{~mol} / \mathrm{l} \mathrm{PBS}$ containing horseradish peroxidase-conjugated rabbit anti-goat IgG (1:500; cat. no. 7074; Cell Signaling Technology, Inc.) for $2 \mathrm{~h}$ at room temperature, followed by development with $0.003 \% \mathrm{H}_{2} \mathrm{O}_{2}$ and $0.03 \%$ 3,30-diaminobenzidine in $0.05 \mathrm{~mol} / \mathrm{l}$ Tris-HCl ( $\mathrm{pH}$ 7.6). Immunohistochemistry for each sample was repeated three times. Slides were then developed with DAB and counterstained with hematoxylin (room temperature, 2 min). The slides were evaluated using a light microscope.

Crystal violet assay. BXPC3 and Suit 2 cells (1,000 cells/well) were cultured for 14 days in the incubator as detailed above. Then, the cells were examined using $1 \%$ crystal violet staining solution at room temperature for $5 \mathrm{~min}$. Following extensive washing, cells were imaged, dissolved using 1\% SDS solution and the optical density (OD) was measured at $600 \mathrm{~nm}$. The experiments were repeated three times. In the AKT inhibitor treatment group, cells were treated with the inhibitor of Akt (AZD5363; MEC, Shanghai, China; $10 \mathrm{nM}$ ) for $24 \mathrm{~h}$.

Over-expression of KrasV12 in NIH3T3 cells. The coding sequence of KrasV12 was inserted into the pBabe vector. The helper plasmid and KrasV12 expression vector were co-transfected into $293 \mathrm{~T}$ cells to produce the retrovirus. The supernatant of the $293 \mathrm{~T}$ cells was collected and added to the NIH3T3 culture and incubated overnight. Cells were selected with puromycine. The resistant cells were collected and examined the expression of exogenous KrasV12 using the antibody for HA tag.

Boyden chamber assay. The upper chamber was loaded with BXPC 3 and Suit 2 cells $\left(2 \times 10^{5}\right)$ and $0.05 \mathrm{ml}$ DMEM (1\% FBS), and $0.152 \mathrm{ml} \mathrm{DMEM} \mathrm{(10 \%} \mathrm{FBS)} \mathrm{was} \mathrm{placed} \mathrm{in} \mathrm{the} \mathrm{lower}$ chamber. Cells were incubated for $12 \mathrm{~h}$. Then, the migratory cells were examined using hematoxylin and eosin staining at room temperature for $3 \mathrm{~min}$. The migratory cells were quantified under an inverted microscope (Olympus Corporation, Tokyo, Japan).

Anchorage-independent growth assay. For the soft agar assay, 5,000 cells/well were suspended in the upper layer $(0.35 \%$ agarose and 10\% FBS in DMEM) in 6-well plates. The plates were coated with a bottom layer $(0.5 \%$ agarose and $10 \%$ FBS in DMEM). After 14 days of incubation, the colonies were counted and measured. All the experiments were performed at least three times.

PDAC mouse model (Pdx-Cre; Loxp-Stop-Loxp KrasG12D). Pdx-Cre; Loxp-Stop-Loxp KrasG12D mice were obtained by crossing the Pdx-Cre mice with the Loxp-Stop-Loxp KrasG12Dmice. Pdx-Cre-mediated recombination removes the transcriptional STOP cassette present in the K-RasG12D allele to allow bicistronic expression of K-RasG12D and induced the PanIN formation 8 month after birth. c57 mice were bred in house at Fujian Medical University (Fuzhou,
China). A total of three 8-week old male mice (body weight $\sim 20-22 \mathrm{~g}$ ) were included in each group. There were two groups in total. Mice were housed at $25^{\circ} \mathrm{C}$ with a 12 -h light/dark cycle with sufficient food and water. After 8 months (the diameter of the tumors was $\sim 1 \mathrm{~mm}$ ), the mice were sacrificed with anesthesia (isoflurane) and the pancreatic tissues obtained.

Tumorigenesis assay. The pcDNA6 plasmids containing the coding sequence for the luciferase gene were transfected into the BXPC3 cells using Lipofectamine 2000 (Invitrogen; Thermo Fisher Scientific, Inc.) according to the manufacturer's protocols. Then, the expression of GOLPH2 was knocked down in BXPC3 cells (BXPC3/si GOLPH2). The cells $\left(1 \times 10^{6}\right)$ were subcutaneously injected into the mice. There were two groups, and four nude mice in each group (SLACK, Shanghai, $\sim 20 \mathrm{~g}$ per mouse). The mice were kept in the SPF housing at $25^{\circ} \mathrm{C}$ with a 12 -h light/dark cycle with sufficient food and water. The growth of the tumor cells was monitored by an in vivo imaging system (PerkinElmer, Inc., Waltham, MA, USA) after administration of the luciferin, the substrate for the luciferase. The tumor cells were traced by the luciferase gene which metabolized luciferin and generated photons. The photons were detected by the CCD camera and the survival rates of the mice analyzed.

Statistical analysis. All data are presented as the mean \pm standard deviation, and statistical comparisons were performed using Student's t-test. Multiple comparisons regarding cell growth and migration were performed using One- or two-way analysis of variance followed by Bonferroni's post hoc test was used for multiple comparisons of data with normal distribution and equal variance (D'Agostino-Pearson omnibus normality test). SPSS software, version 15.0 (SPSS, Inc., Chicago, IL, USA) was used to analyze data and each experiment was repeated at least three times. $\mathrm{P}<0.05$ was considered to indicate a statistically significant difference.

\section{Results}

GOLPH2 is upregulated in PDAC clinical samples. To determine the mRNA level of GOLPH2 in pancreatic cancer tissue, RT-qPCR was performed. Compared with the adjacent normal tissues, GOLPH2 was observed to be upregulated in PDAC clinical samples (Fig. 1A). Furthermore, the immunohistochemistry staining revealed that the protein level of GOLPH2 was significantly increased in tumor tissues (Fig. 1B), which was consistent with the observations from Fig. 1A. The protein level of GOLPH2 in 5 PDAC tissues and paired adjacent normal tissues was examined. Increased GOLPH2 protein levels were observed in $80 \%$ (4 out of 5) of the PDAC tissues (Fig. 1C). Notably, the majority of non-cancerous samples demonstrated a low protein expression of GOLPH2, however the mRNA levels of GOLPH2 were detectable (Fig. 1A and C). These data indicated increased expression levels of GOLPH2 in pancreatic cancer, compared with normal healthy tissue.

GOLPH2 promotes growth and motility of pancreatic cancer cells. To further study the role of GOLPH2 in the progression of pancreatic cancer, GOLPH2 was overexpressed in BXPC3 
A

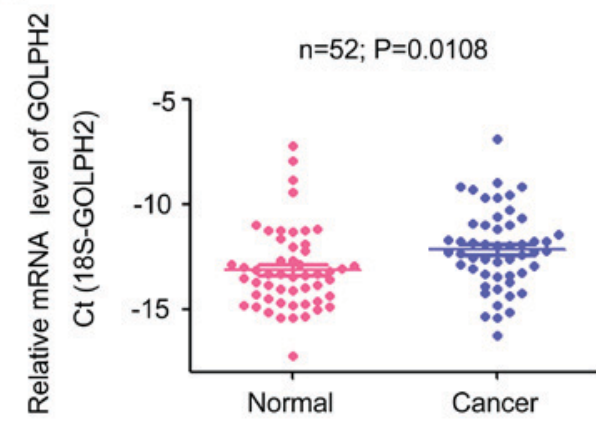

C

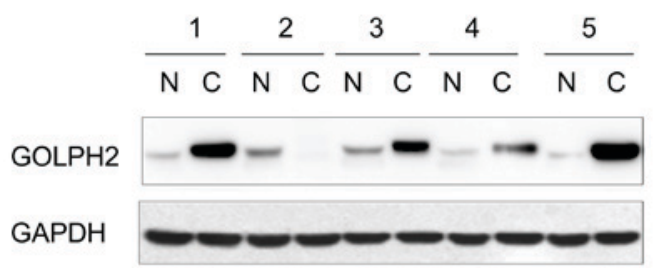

B

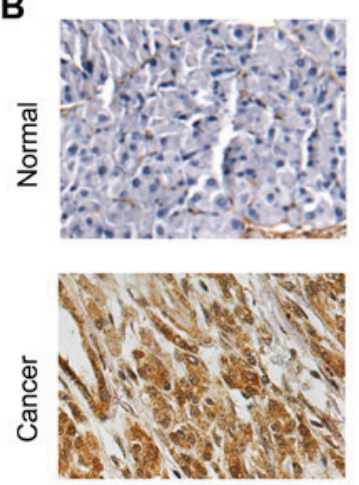

Figure 1. Expression of GOLPH2 is upregulated in PDAC clinical samples. (A) The mRNA level of GOLPH2 was increased in PDAC samples. GOLPH2 mRNA levels in 52 PDAC samples and 52 paired adjacent normal tissues were examined using reverse transcription-quantitative polymerase chain reaction. The GOLPH2 expression was normalized to that of $18 \mathrm{~S}$. Data was obtained from triplicate experiments. (B) Immunohistochemistry staining revealed upregulation of GOLPH2 protein in PDAC samples compared with paired adjacent normal tissues. (C) Western blotting analysis examined the protein level of GOLPH2 in PDAC samples and paired adjacent normal tissues. GOLPH2, Golgi protein-73; PDAC, pancreatic ductal adenocarcinoma.
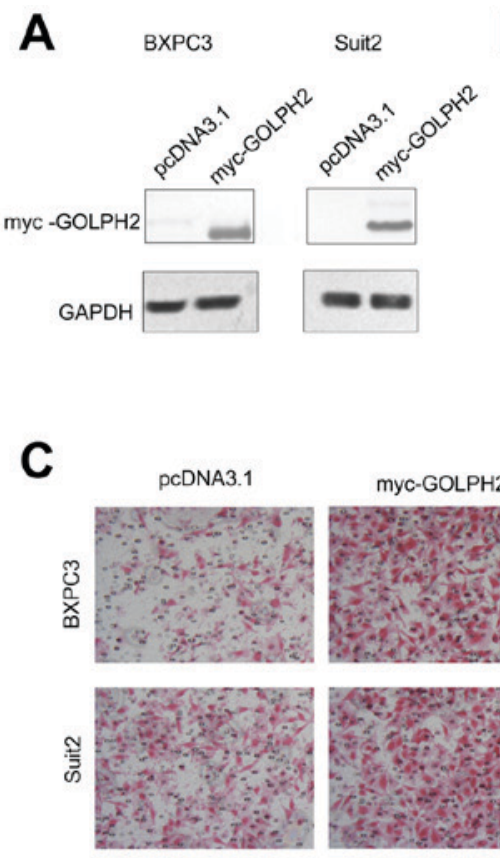
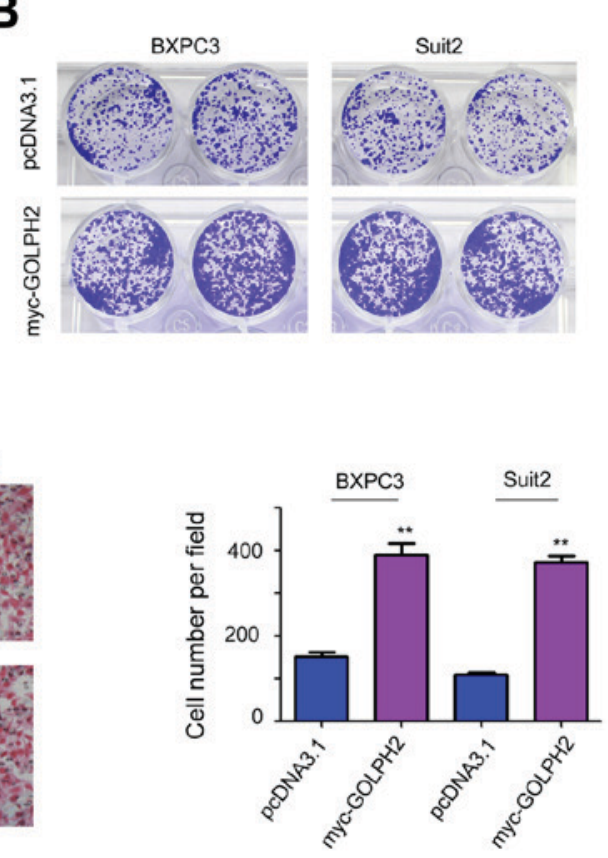

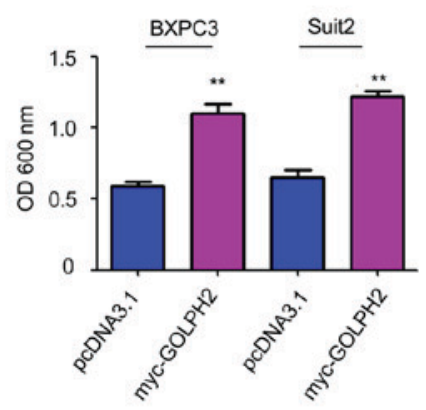

Figure 2. GOLPH2 promotes growth and migration of pancreatic ductal adenocarcinoma cells. (A) Forced expression of myc-tagged GOLPH2 in BXPC3 and Suit2 cells. BXPC3 and Suit2 cells were transfected with pcDNA3.1 vector or the GOLPH2 expression vector, and selected with G418. G418-resistant cells were pooled and overexpression of GOLPH2 was verified by western blot analysis. (B) Colony formation assay to examine the effects of GOLPH2 on the growth of BXPC3 and Suit2 cells by crystal violet staining. (C) Forced expression of GOLPH2 promoted the migration of BXPC3 and Suit2 cells detected using a Boyden chamber assay. ${ }^{* *} \mathrm{P}<0.01$ vs. pcDNA3.1. GOLPH2, Golgi protein-73; pcDNA3.1, control expression vector; myc-GOLPG2, myc-tagged pcDNA3.1 GOLPH2 expression vector; OD, optical density.

and Suit2 cells (Fig. 2A). Then, the function of increased GOLPH 2 on the growth and motility of BXPC 3 and Suit2 cells was examined. As presented in Fig. 2B, GOLPH2 significantly promoted cell growth. In addition, GOLPH2 enhanced cell migratory activity of pancreatic cancer cells in the Boyden chamber assay (Fig. 2C). Collectively, these observations demonstrated that GOLPH2 promoted cell growth and migration in pancreatic cancer cells.

The effects of exogenous GOLPH2 on the growth and migration of the pancreatic cancer cells led to examination of 

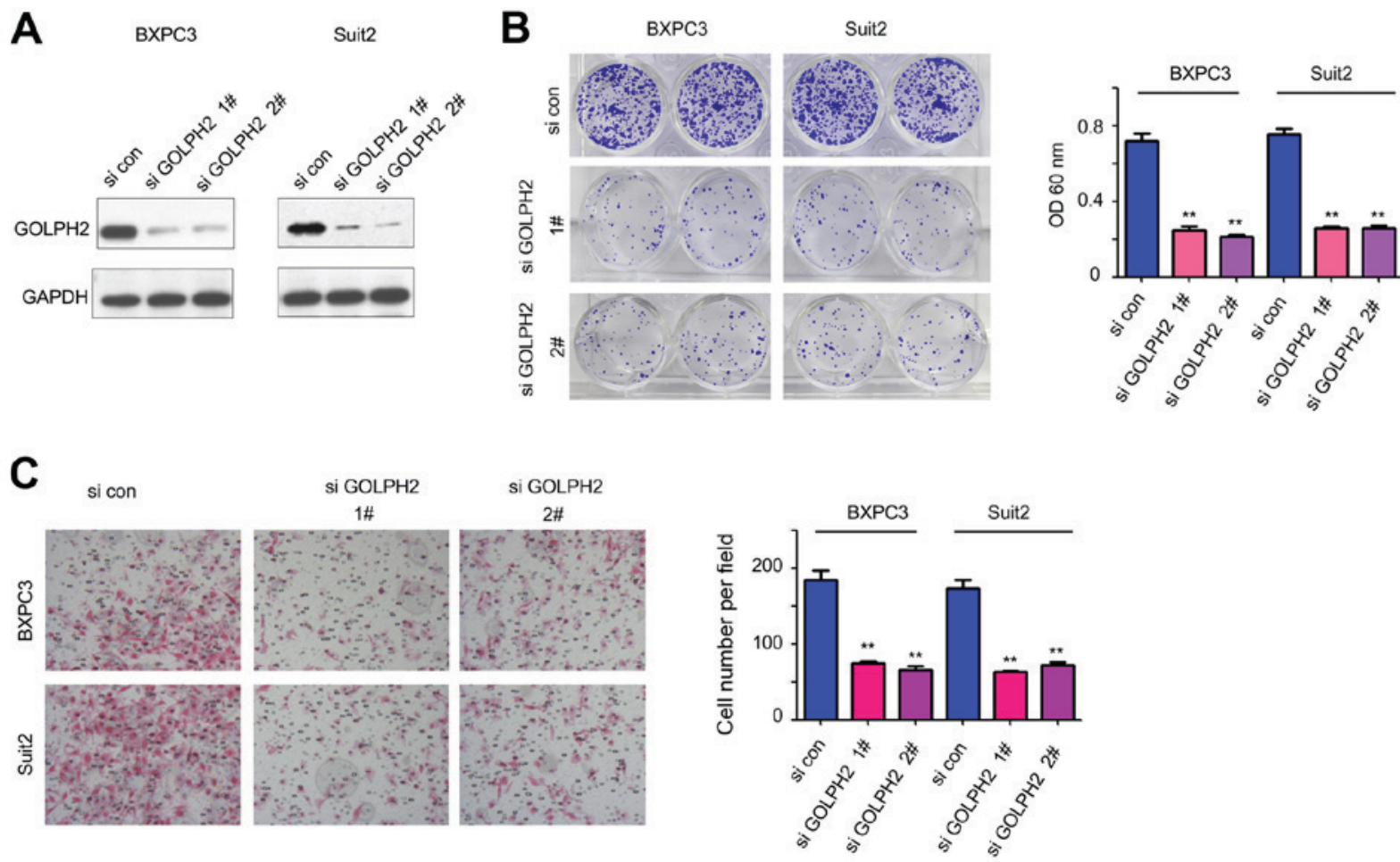

Figure 3. Downregulation of GOLPH2 impairs the growth and migratory activity of BXPC3 and Suit2 cells. (A) Expression of GOLPH2 in BXPC3 and Suit2 cells was downregulated by short hairpin RNA interference. (B) Knockdown of GOLPH2 expression inhibited the (B) colony formation and (C) migratory activity of BXPC 3 and Suit 2 cells, detected by crystal violet staining and Boyden chamber assay, respectively. ${ }^{* *} \mathrm{P}<0.01$ vs. si con. GOLPH2, Golgi protein-73; si con, small interfering RNA control; si GOLPH2, RNAi lenti-virus targeting GOLPH2; OD, optical density.

the role of endogenously expressed GOLPH2. The expression of endogenous GOLPH2 was knocked down using two independent siRNA sequences (Fig. 3A). Knockdown of GOLPH2 expression in BXPC3 and Suit2 cells inhibited cell growth, as demonstrated by the crystal violet staining (Fig. 3B). In addition, downregulation of GOLPH2 attenuated the motility of pancreatic cancer cells (Fig. 3C). These observations were consistent with the findings in the gain-of-function assay, and further confirmed the tumor-promoting functions of GOLPH2 in pancreatic cancer.

GOLPH2 binds with Akt and enhances Akt activity. To explore the underlying molecular mechanism, mass spectrometry was used to identify the binding proteins of GOLPH2 (data not shown). Akt was demonstrated to be the most efficient candidate. The present study firstly examined the effects of GOLPH2 on the Akt signaling. Inhibiting GOLPH2 expression impaired the phosphorylation of Akt Thr473 (Fig. 4A). Furthermore, downregulation of GOLPH2 promoted the expression of Bax, and decreased the protein levels of Cyclin D1 and Bcl2, which are downstream effectors of Akt signaling (Fig. 4B). The inhibitor of Akt (AZD5363, MEC, $10 \mathrm{nM}$, treated for $24 \mathrm{~h}$ ) abolished the cell growth induced by GOLPH2 (Fig. 4C). These findings demonstrated the regulation of Akt signaling by GOLPH2. The interaction between GOLPH 2 and Akt was further confirmed by a GST-pull down assay and immunoprecipitation assay. As presented in Fig. 4D, the interaction between GST-Akt and GOLPH2 was detected in BXPC3 cells. Furthermore, the endogenously expressed GOLPH2 was demonstrated to form a complex with Akt (Fig. 4E). Collectively, these results suggested that GOLPH2 interacted with and positively regulated Akt signaling.

GOLPH2 is a downstream gene of Kras signaling and promotes transformation of normal pancreatic cells. The oncogenic functions of GOLPH2 in pancreatic cancer cells led to an examination of its role in the early stage of pancreatic carcinogenesis. The present study used a pancreatic cancer mouse model Pdx-Cre; KrasG12D in which pancreatic intraepithelial neoplasia (PanINs), precursors of PDAC, were formed at the age of 10 months (Fig. 5A). Next, the expression of GOLPH2 in the PanINs was examined. Upregulation of GOLPH2 was observed in PanINs, suggesting the regulation of GOLPH2 by the oncogenic Kras signaling (Fig. 5B). Consistent with these observations, overexpression of KrasV12 in NIH3T3 and normal pancreatic cells HPDE upregulated the expression of GOLPH2 (Fig. 5C). Furthermore, knocking down the expression of GOLPH2 abolished cell transformation induced by KrasV12 in the anchorage-independent growth assay (Fig. 5D), indicating the important function of GOLPH2 in the tumorigenesis of pancreatic cancer.

Knockdown of GOLPH2 expression inhibits tumorigenesis of pancreatic cancer cells in nude mice. Next, the present study investigated the effects of GOLPH2 on the tumorigenesis of pancreatic cancer cells. BXPC3 cells underwent forced expression of the luciferase gene, which enabled the tracing of tumor cells in vivo. Knocking down the expression of GOLPH2 inhibited the tumor growth in the nude mice, which was demonstrated 
A

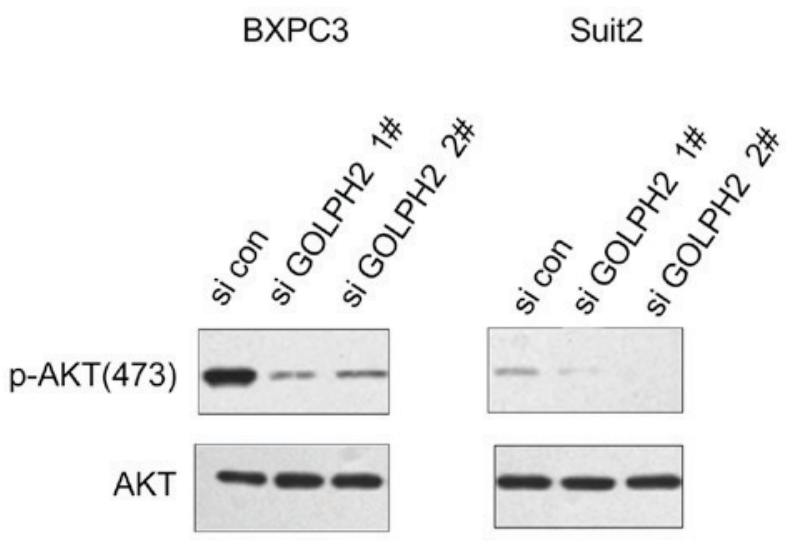

C

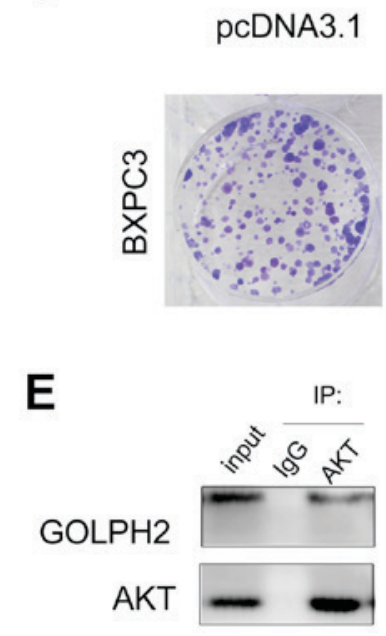

B

BXPC3

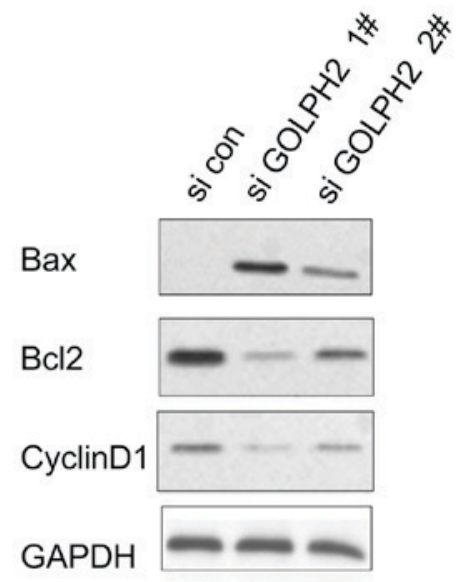

D

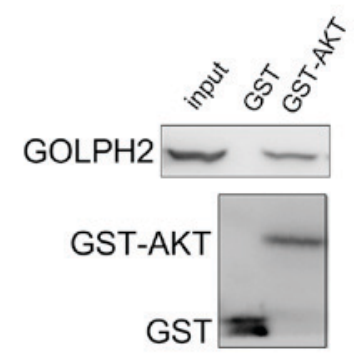

Figure 4. GOLPH2 activates Akt signaling in BXPC3 and Suit2 cells. (A) Knocking down the expression of GOLPH2 decreased the phosphorylation level of Akt. (B) Knocking down the expression of GOLPH2 inhibited the expression of target genes downstream of Akt signaling. (C) An Akt inhibitor abolished the growth advantage of BXPC3 cells induced by GOLPH2. (D) GST-pull down assay to verify the interaction between GOLPH2 and Akt. (E) Interaction between Akt and GOLPH2 in BXPC3 cells was demonstrated by an immunoprecipitation assay. GOLPH2, Golgi protein-73; si con, small interfering RNA control; si GOLPH2, RNAi lenti-virus targeting GOLPH2; p, phosphorylated; Akt, protein kinase B; pcDNA3.1, control expression vector; myc-GOLPH2, myc-tagged pcDNA3.1 GOLPH2 expression vector; Bcl-2, B-cell lymphoma; Bax, Bcl-2 associated X, apoptosis regulator; IP, immunoprecipitation; IgG, immunoglobin G.

by the weaker photon counts (Fig. 6A and B). Furthermore, down-regulation of GOLPH2 improved the survival of the nude mice (Fig. 6C). These results further emphasized the oncogenic role of GOLPH2 in pancreatic cancer.

\section{Discussion}

The present study demonstrated that expression levels of GOLPH2 were increased in pancreatic cancer tissues compared with adjacent normal tissue. The expression level of GOLPH2 in the serum of the pancreatic cancer patients was not examined, therefore further investigation of the expression of GOLPH2 in serum of patients would clarify the importance of GOLPH2 in the diagnosis of this disease. Furthermore, GOLPH2 was demonstrated to promote the growth and motility of cancer cells in the present study. Notably, GOLPH2 interacted with Akt, promoted its phosphorylation and regulated the expression of a panel of genes involved in cell growth and apoptosis, which explained the role of GOLPH2 in pancreatic cancer. In addition, GOLPH2 expression was revealed to be induced by the oncogenic Kras signaling and transformed the normal pancreatic cells. The present study revealed the oncogenic role of GOLPH2 in pancreatic cancer, and suggested that GOLPH2 may act as a potential target for treatment.

A previous study identified the progression from pancreatitis to PanIN, followed by development of PDAC (14). It has previously been demonstrated that inflammation is a high-risk factor for the development of PDAC (15). Previous studies demonstrated that the expression of GOLPH2 is induced under inflammatory conditions (16). The present study did not examine the expression of GOLPH 2 in the pancreatitis tissue, however it is possible that pancreatitis tissues may demonstrate an upregulation of GOLPH2, and further investigation is required to verify this. Therefore, GOLPH2 may be upregulated at the early stage of tumorigenesis. 
A

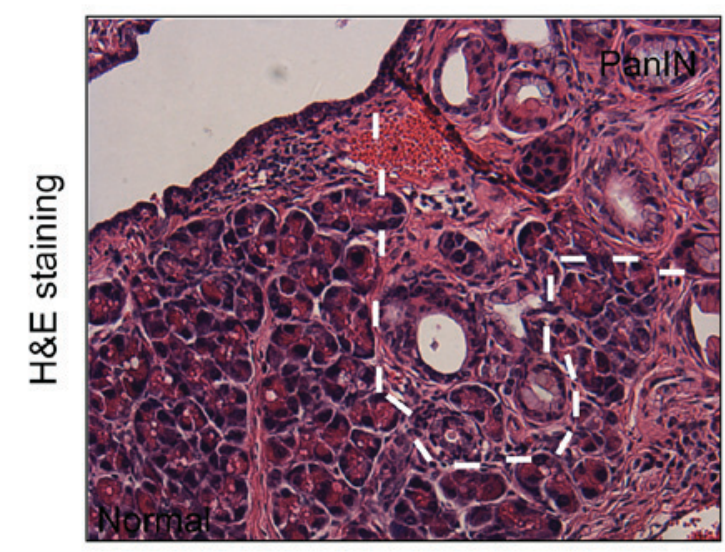

B

\section{GOLPH2}

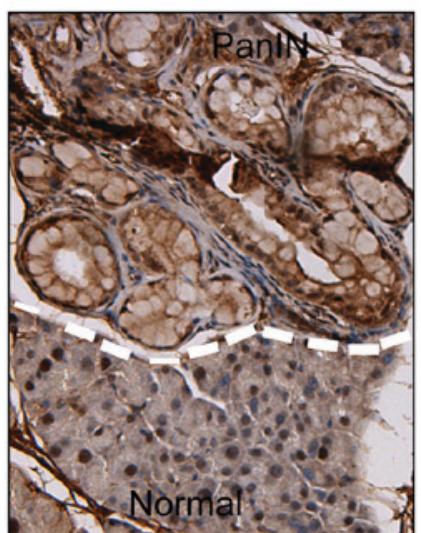

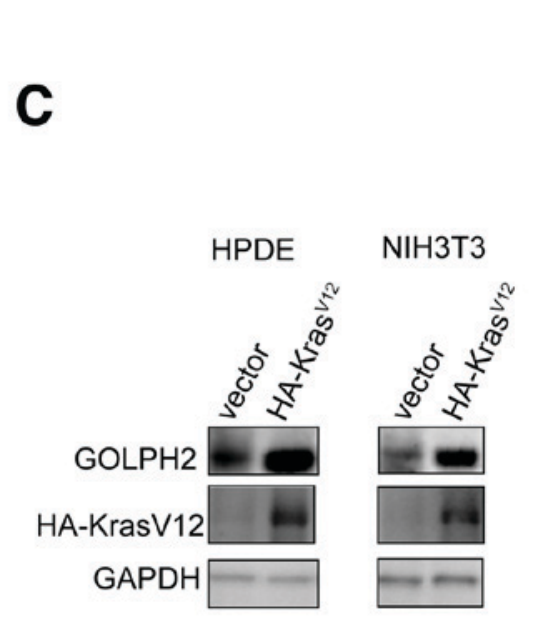
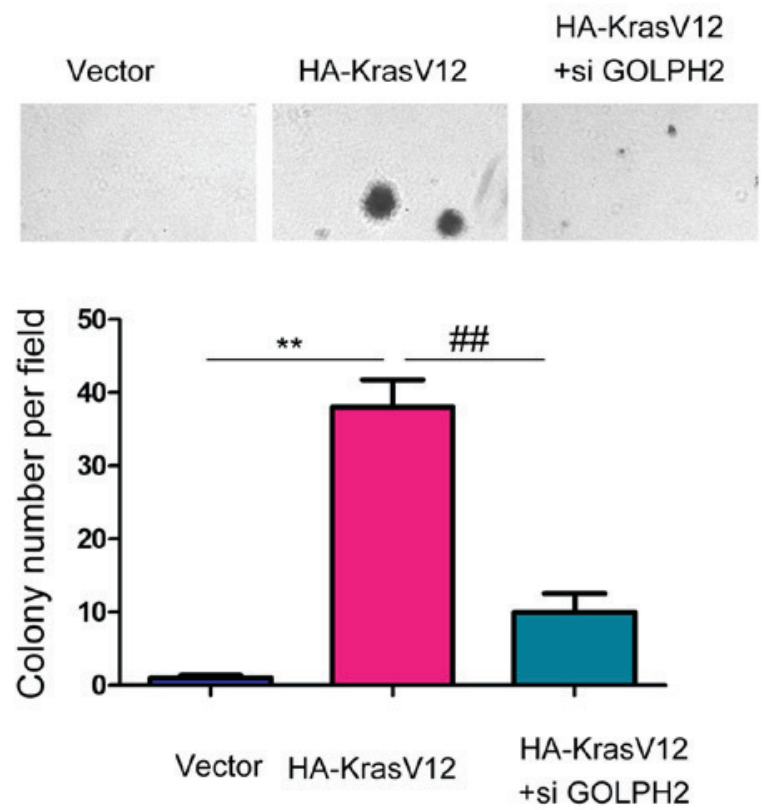

Figure 5. Oncogenic Ras induces the expression of GOLPH2. (A) H\&E staining of pancreatic tissues derived from PDX-cre; LSL-KrasG12D mice. (B) Expression of GOLPH2 was upregulated in PanIN tissues examined by immunohistochemistry staining. (C) Exogenously expressed KrasV12 with HA tag (HA-KrasV12) induced the expression of GOLPH2 in HPDE and NIH3T3 cells. (D) Knockdown of the expression of GOLPH2 impaired the malignant transformation of NIH3T3 cells driven by KrasV12. ${ }^{* *} \mathrm{P}<0.01 ;{ }^{* \#} \mathrm{P}<0.01$. GOLPH2, Golgi protein-73; si GOLPH2, RNAi lenti-virus targeting GOLPH2; H\&E, hematoxylin and eosin; PDX-cre LSL-KrasG12D, Pdx-Cre-mediated recombination to remove transcriptional STOP cassette present in the K-RasG12D allele to allow bicistronic expression of K-RasG12D; PanIN, pancreatic intraepithelial neoplasia.; HA, tag of the fusion protein.

A

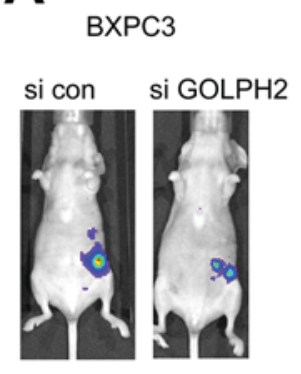

B

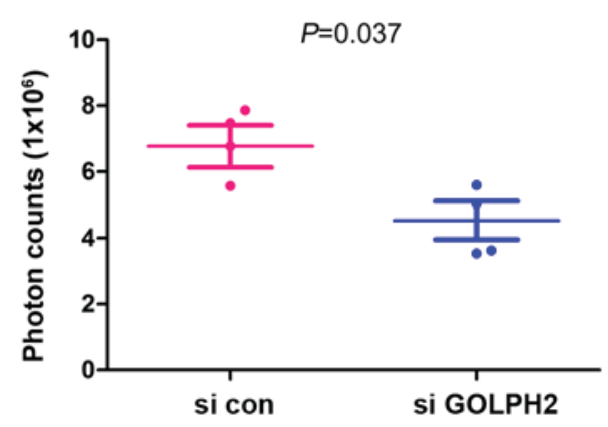

C

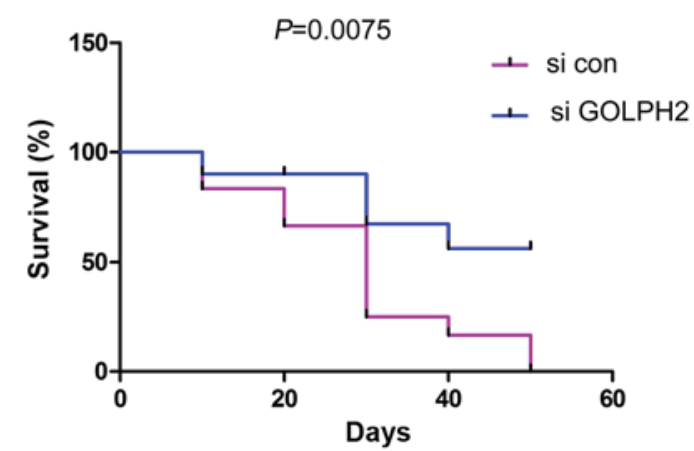

Figure 6. Knocking down the expression of GOLPH2 inhibits the tumorigenesis of BXPC-3 cells. (A) Representative image and (B) quantification of photon counts to demonstrate tumors formed by BXPC-3/si con cells and BXPC-3/si GOLPH2 cells. The tumor volume was reflected by the photon counts examined by the image system. (C) Survival rate of the mice bearing tumors formed by BXPC-3/si con cells and BXPC-3/si GOLPH2 cells. GOLPH2, Golgi protein-73; si con, small interfering RNA control; si GOLPH2, RNAi lenti-virus targeting GOLPH2. 
A previous study identified the spatial regulation of Akt signaling, and GOLPH3 has been reported to regulate Akt-mechanistic target of rapamycin signaling in the Golgi apparatus (13). The results of the aforementioned study, in combination with the results of the present study, reveal the mechanism of the spatial regulation of Akt signaling in the Golgi apparatus. Considering the importance of GOLPH2 in Akt signaling, further experiments would involve elucidating the physiological outcomes and specific molecular mechanisms underlying the regulation of GOLPH2.

In conclusion, the results of the study revealed the tumor-promoting role of GOLPH2 in the progression of pancreatic cancer. GOLPH2 may serve as the therapeutic target and as a tumor marker for PDAC. Further study using the GOLPH2 mouse model will elucidate the novel functions of GOLPH2.

\section{Acknowledgements}

The present study was supported by the Natural Science Foundation of Fujian Province (grant no. 2013J05048) and the PhD Research Startup Foundation of Fujian Medical University (grant no. 2011BS001).

\section{References}

1. Siegel RL, Miller KD and Jemal A: Cancer statistics, 2015. CA Cancer J Clin 65: 5-29, 2015

2. Wei S, Dunn TA, Isaacs WB, De Marzo AM and Luo J: GOLPH2 and MYO6: Putative prostate cancer markers localized to the golgi apparatus. Prostate 68: 1387-1395, 2008.

3. Riener MO: Diagnosis of tumours of the liver and the biliary tract: New tissue and serum markers. Pathologe 2 (32 Suppl): S304-S309, 2011 (In German).

4. Riener MO, Stenner F, Liewen H, Hellerbrand C, Bahra M and Kristiansen G: Alpha-fetoprotein and serum golgi phosphoprotein 2 are equally discriminative in detecting early hepatocellular carcinomas. Hepatology 50: 326, 2009.

5. Fritzsche FR, Riener MO, Dietel M, Moch H, Jung K and Kristiansen G: GOLPH2 expression in renal cell cancer. BMC Urol 8: 15, 2008.
6. Zhang F, Gu Y, Li X, Wang W, He J and Peng T: Up-regulated golgi phosphoprotein 2 (GOLPH2) expression in lung adenocarcinoma tissue. Clin Biochem 43: 983-991, 2010.

7. Kristiansen G, Fritzsche FR, Wassermann K, Jäger C, Tölls A, Lein M, Stephan C, Jung K, Pilarsky C, Dietel M and Moch H: GOLPH2 protein expression as a novel tissue biomarker for prostate cancer: Implications for tissue-based diagnostics. Br J Cancer 99: 939-948, 2008.

8. Laurent-Puig P, Lievre A and Blons H: Mutations and response to epidermal growth factor receptor inhibitors. Clin Cancer Res 15: 1133-1139, 2009.

9. Sharma N, Nanta R, Sharma J, Gunewardena S, Singh KP, Shankar S and Srivastava RK: PI3K/AKT/mTOR and sonic hedgehog pathways cooperate together to inhibit human pancreatic cancer stem cell characteristics and tumor growth. Oncotarget 6: 32039-32060, 2015.

10. Buck E, Eyzaguirre A, Haley JD, Gibson NW, Cagnoni P and Iwata KK: Inactivation of Akt by the epidermal growth factor receptor inhibitor erlotinib is mediated by HER-3 in pancreatic and colorectal tumor cell lines and contributes to erlotinib sensitivity. Mol Cancer Ther 5: 2051-2059, 2006.

11. Xu C, Hu DM and Zhu Q: eEF1A2 promotes cell migration, invasion and metastasis in pancreatic cancer by upregulating MMP-9 expression through Akt activation. Clin Exp Metastasis 30: 933-944, 2013.

12. Zeng Z, Lin H, Zhao X, Liu G, Wang X, Xu R, Chen K, Li J and Song L: Overexpression of GOLPH3 promotes proliferation and tumorigenicity in breast cancer via suppression of the FOXO1 transcription factor. Clin Cancer Res 18: 4059-4069, 2012.

13. Livak KJ and Schmittgen TD: Analysis of relative gene expression data using real-time quantitative PCR and the 2(-Delta Delta $\mathrm{C}(\mathrm{T})$ ) method. Methods 25: 402-408, 2001

14. Bardeesy N and DePinho RA: Pancreatic cancer biology and genetics. Nat Rev Cancer 2: 897-909, 2002.

15. Guerra C, Collado M, Navas C, Schuhmacher AJ, Hernández-Porras I, Cañamero $M$, Rodriguez-Justo $M$, Serrano $M$ and Barbacid M: Pancreatitis-induced inflammation contributes to pancreatic cancer by inhibiting oncogene-induced senescence. Cancer cell 19: 728-739, 2011.

16. Wang F, Long Q, Gong Y, Hu L, Zhang H, Oettgen $\mathrm{P}$ and Peng T: Epithelium-Specific ETS (ESE)-1 upregulated GP73 expression in hepatocellular carcinoma cells. Cell Biosci 4: 76, 2014.

This work is licensed under a Creative Commons Attribution-NonCommercial-NoDerivatives 4.0 International (CC BY-NC-ND 4.0) License. 